\title{
Longitudinal Focusing and Cooling of a Molecular Beam
}

\author{
Floris M. H. Crompvoets, Rienk T. Jongma, Hendrick L. Bethlem, André J. A. van Roij, and Gerard Meijer \\ FOM-Institute for Plasmaphysics Rijnhuizen, P.O. Box 1207, NL-3430 BE Nieuwegein, The Netherlands \\ and Department of Molecular and Laser Physics, University of Nijmegen, Toernooiveld 1, NL-6525 ED Nijmegen, The Netherlands
}

(Received 6 May 2002; published 13 August 2002)

\begin{abstract}
A neutral polar molecule experiences a force in an inhomogeneous electric field. This electric field can be designed such that a beam of polar molecules is exposed to a harmonic potential in the forward direction. In this potential the longitudinal phase-space distribution of the ensemble of molecules is rotated uniformly. This property is used to longitudinally focus a pulsed beam of ammonia molecules and to produce a beam with a longitudinal velocity spread of $0.76 \mathrm{~m} / \mathrm{s}$, corresponding to a temperature of $250 \mu \mathrm{K}$.
\end{abstract}

DOI: 10.1103/PhysRevLett.89.093004

PACS numbers: 33.80.Ps, 33.55.Be, 39.10.+j

Currently there is great interest in the physics of cold molecules, in general [1,2], and in the physics of cold polar gases, in particular. The latter originates, for instance, from the anticipated importance of the anisotropic interaction between electric dipoles on the formation of a molecular Bose-Einstein condensate [3], from the possibility to use single component cold polar gases for the observation of the superfluid transition [4], and from the possibility to use arrays of trapped polar molecules for quantum computation [5]. One of the methods that has recently been successful in producing cold molecules uses time-varying electric fields to decelerate pulsed beams of polar molecules [6-8]. The thus produced molecular beams with their tunable laboratory velocity, i.e., with their tunable de Broglie wavelength, offer fascinating perspectives for molecule optics $[9,10]$ as well as for collision studies [11]. The longitudinal velocity spread of these decelerated beams corresponds to a longitudinal temperature of several $\mathrm{mK}$, only slightly higher than the lowest temperature $\mathrm{He}$ beam that has been reported [12]. Bunches of these slow and cold molecules can be stored in an electrostatic storage ring [13] or decelerated to a near standstill and trapped in an electrostatic quadrupole trap $[14,15]$.

In the molecular beam experiments, transverse collimation and focusing can be achieved by using electrostatic lenses, e.g., hexapole lenses and alternate gradient lenses for polar molecules in low-field seeking states and in highfield seeking states, respectively. To further increase the number density at a given point along the molecular beam axis, the molecular beam needs to be focused in the forward direction as well. Such a "spatial bunching" of the molecular beam is obviously advantageous for collision studies and can, for instance, be used to optimize the number of molecules loaded from the decelerated beam into the electrostatic trap [15]. Spatial bunching is also highly desirable inside an electrostatic storage ring [13], as it will allow observation of more round trips. To further reduce the longitudinal temperature of the beam, the forward velocity distribution needs to be "focused." Such a "velocity bunching" keeps a package of decelerated mol- ecules together for a longer period of time. This is highly desirable in molecular beam diffraction studies, for instance, as it increases the phase contrast.

In this Letter we experimentally demonstrate the operation principle of a buncher for neutral polar molecules. In the buncher, a beam of polar molecules is exposed to a harmonic potential in the forward direction, i.e., along the molecular beam axis. This results in a uniform rotation of the longitudinal phase-space distribution of the ensemble of molecules. By switching the buncher on and off at the appropriate times, it can be used either to produce a narrow spatial distribution at a certain position downstream from the buncher or to produce a narrow velocity distribution. A reduction in the width of the spatial distribution is accompanied by an increase in the width of the velocity distribution and vice versa, as the phase-space density remains constant in this process [16]. In the field of charged particle beams, where bunching elements are routinely used, these operations are commonly referred to as rebunching and bunch rotation, respectively [17]. Proposals have been put forward to use time-varying magnetic fields for longitudinal focusing of atoms [18,19] and neutrons [20], both in real space and in velocity space. For atoms, where bunch rotation is often referred to as " $\delta$-kick cooling," these operations have recently been experimentally demonstrated [21,22].

The molecular beam machine that is used for the longitudinal focusing and cooling experiments is schematically depicted in Fig. 1. A beam of deuterated ammonia is formed by expanding a mixture of less than $1 \%{ }^{14} \mathrm{ND}_{3}$ seeded in xenon through a pulsed valve into vacuum. The valve is operated at a temperature of $200 \mathrm{~K}$, resulting in an average velocity of the molecular beam of approximately $285 \mathrm{~m} / \mathrm{s}$. The relative velocity spread in the beam is typically $20 \%$, corresponding to a longitudinal temperature of $1.5 \mathrm{~K}$. Because of adiabatic cooling in the expansion, only the lowest rotational levels in the vibrational and electronic ground states are populated in the beam. Roughly $60 \%$ of all the $\mathrm{ND}_{3}$ molecules in the beam reside in the $|J, K\rangle=$ $|1,1\rangle$ level, the ground-state level for para-ammonia 


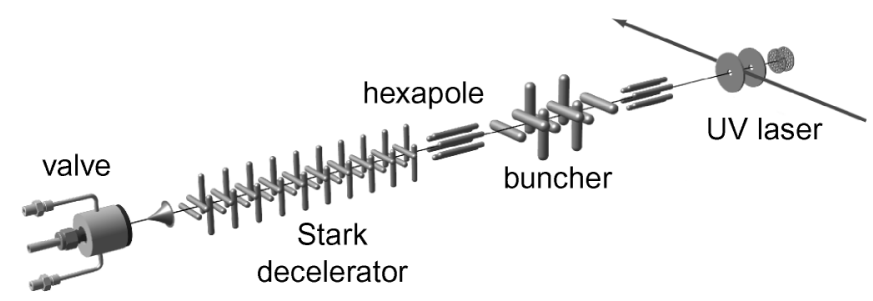

FIG. 1. Scheme of the experimental setup. A pulsed beam of ammonia molecules is decelerated and passes through a buncher. The arrival time distribution of the package of molecules at the laser interaction zone is recorded using a UV-laser based ionization detection scheme.

molecules. For the experiments reported here, only ammonia molecules in the low-field seeking levels of the upper component of this inversion doublet are used. The molecular beam passes through a skimmer and enters a so-called Stark decelerator, mounted in a second vacuum chamber. The $35 \mathrm{~cm}$ long decelerator consists of an array of 64 equidistant electric field stages. When the ammonia molecules in low-field seeking levels enter a region of high electric field (up to $90 \mathrm{kV} / \mathrm{cm}$ ), they will gain Stark energy. This gain in Stark energy ("potential" energy) is compensated by a loss in kinetic energy. If the electric field is greatly reduced before the molecules have left this region, they will not regain the lost kinetic energy. This process is repeated by letting the molecules pass through the array of electric field stages, which are switched synchronously with the arrival of the package of decelerating molecules. The process in the Stark decelerator, the equivalent of a charged particle linear accelerator, can be viewed as slicing a bunch of molecules with both a narrow spatial distribution and a narrow velocity distribution (determined by the settings of the decelerator) out of the original beam, and decelerating these to arbitrarily low absolute velocities. In this process the phase-space density remains constant [7], and one can thus translate the high phase-space densities from the moving frame of the molecular beam to the laboratory frame. A detailed description of the molecular beam machine and of the deceleration of ammonia using time-varying electric fields is given elsewhere [15].

In the experiments reported here, the Stark decelerator is operated such that a $1 \mathrm{~mm}$ long bunch of ammonia molecules with an average forward velocity of $91.8 \mathrm{~m} / \mathrm{s}$ and with a longitudinal velocity spread of about $6.5 \mathrm{~m} / \mathrm{s}$ exits the decelerator. The calculated longitudinal phase-space distribution of this initially prepared molecular beam is shown in Fig. 2 at $t=0 \mathrm{~ms}$. This is the distribution relative to the position in phase space of the "synchronous molecule" [15], at the moment that the decelerator is switched off. At this moment, the synchronous molecule is located on the molecular beam axis ( $z$ axis) $0.6 \mathrm{~mm}$ upstream from the center of the last electric field stage of the decelerator, and is moving with a velocity of $v_{z}=91.8 \mathrm{~m} / \mathrm{s}$ along the $z$ axis. The entrance of the buncher is located some $15 \mathrm{~cm}$ downstream from the exit of the decelerator. A $5 \mathrm{~cm}$ long

093004-2

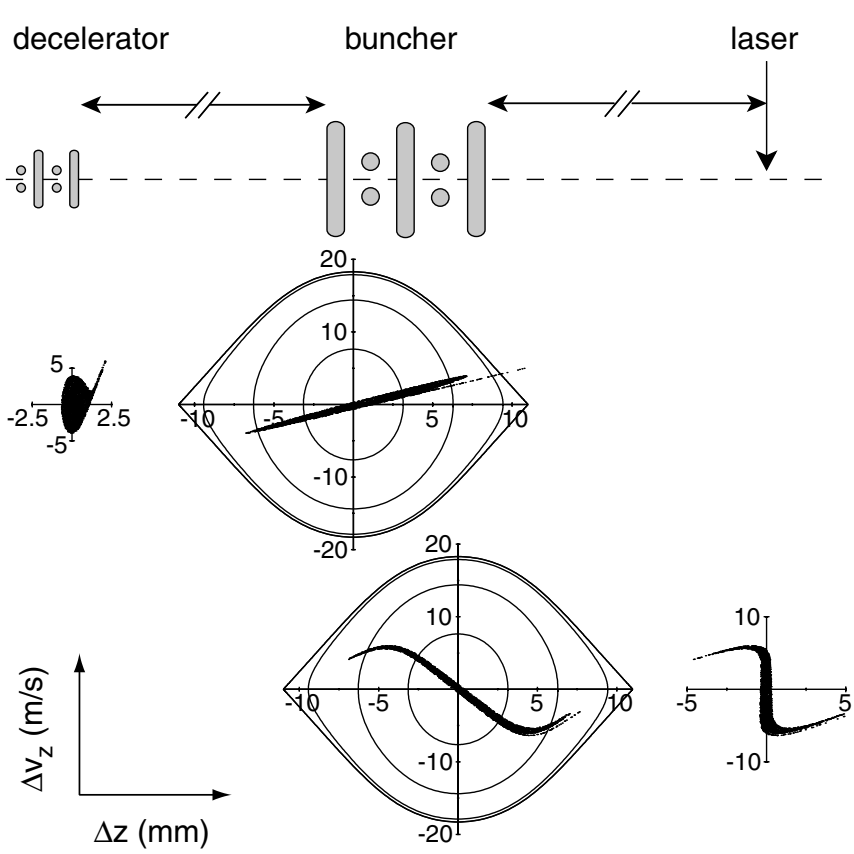

FIG. 2. Expanded view of the end of the decelerator, the buncher, and the detection region. The calculated longitudinal phase-space distribution of the ammonia molecules is given at the exit of the Stark decelerator $(t=0 \mathrm{~ms})$, at the entrance $(t=$ $1.743 \mathrm{~ms})$ and exit $(t=2.100 \mathrm{~ms})$ of the buncher, and in the laser detection region $(t=2.652 \mathrm{~ms})$, relative to the position in phase space of the synchronous molecule.

hexapole is installed between the decelerator and the buncher. The hexapole is switched on for a few tens of $\mu$ s once the package of ammonia molecules is completely inside it; the effective length of the hexapole is thus only a few $\mathrm{mm}$. The hexapole focuses molecules in low-field seeking states in the transverse direction, but it leaves their forward velocity unchanged. In flying from the exit of the decelerator to the buncher, the package of ammonia molecules spreads out along the molecular beam axis. This results in the elongated and tilted distribution in longitudinal phase-space as shown in Fig. 2 for $t=1.743 \mathrm{~ms}$, the time that the buncher is switched on.

The buncher consists of an array of five electric field stages, with a center-to-center distance along the molecular beam axis of $11 \mathrm{~mm}$. Each stage consists of two parallel cylindrical metal rods with a diameter of $6 \mathrm{~mm}$, centered at a distance of $10 \mathrm{~mm}$. One of the rods is connected to a positive and the other to a negative switchable high-voltage power supply. Alternating stages are connected to each other. As the electric field close to the electrodes is higher than that on the molecular beam axis, molecules in lowfield seeking states will experience a force focusing them towards this axis. This focusing occurs only in the plane perpendicular to the electrodes. By alternately orienting the electric field stages horizontally and vertically, molecules are focused in either transverse direction.

When the synchronous molecule is exactly in between the first and second electric field stages of the buncher, the 
high voltage on the odd numbered electric field stages is switched on. The synchronous molecule is now on the downward slope of a potential well, and will be accelerated. When the synchronous molecule is $11 \mathrm{~mm}$ farther, on the upward slope of the potential well, it is decelerated again to its original velocity. At this time the high voltage on the odd numbered stages is switched off. Molecules that are originally slightly ahead of the synchronous molecule, i.e., molecules that are originally faster, will spend more time on the upward slope than on the downward slope of the potential well, and are therefore decelerated relative to the synchronous molecule. Molecules that are originally slightly behind the synchronous molecule, i.e., molecules that are originally slower, will spend less time on the upward slope than on the downward slope of the potential well, and are therefore accelerated relative to the synchronous molecule. This process can be repeated, by switching on the high voltage on the even numbered stages when the odd numbered stages are switched off. Evidently, this can be done for a maximum of 3 times in our present buncher. The electric fields in the buncher are designed such that the (series of) potential well(s) experienced by the ammonia molecules along the molecular beam axis is harmonic over an interval of approximately $1 \mathrm{~cm}$ around the minimum of the well. It is noted that the operation principle of the buncher is equivalent to that of the Stark decelerator at a phase angle of $0^{\circ}$ [7].

During the time that the buncher is on, the longitudinal phase-space distribution is rotated in the $\left(z, v_{z}\right)$ plane. The calculated distribution at $t=2.100 \mathrm{~ms}$, the time that the buncher is switched off, is shown in Fig. 2 for the case that a series of three potential wells is used in the buncher and with voltages of $+9.5 \mathrm{kV}$ and $-9.5 \mathrm{kV}$ applied to the electrodes. In this figure, the contours of equal energy for the ammonia molecules in the potential well are shown, relative to the position in phase space of the synchronous molecule. It is seen that the rotation in phase space is uniform, i.e., that the potential is harmonic, near the center and that the rotation is slower farther outward, reaching zero at the separatrix. For optimum performance, the experiment has to be designed such that mainly the harmonic part of the acceptance diagram of the buncher is used. At the time that the buncher is switched off, slow molecules are ahead while fast molecules are lagging behind, leading to a longitudinal spatial focus farther downstream. In the particular situation depicted in Fig. 2, the ammonia beam has a longitudinal focus with a width of about $0.5 \mathrm{~mm}$, some $4.5 \mathrm{~cm}$ after the end of the buncher.

In the experiments the ammonia beam is probed either at a distance of $4.5 \mathrm{~cm}$ or at a distance of $33 \mathrm{~cm}$ downstream from the buncher. In the latter case, a second pulsed hexapole is used to transversely focus the ammonia beam exiting the buncher into the laser detection region. Using a $(2+1)$-resonance enhanced multiphoton ionization scheme with a pulsed laser around $317 \mathrm{~nm},{ }^{14} \mathrm{ND}_{3}$ molecules in the upper component of the $|J, K\rangle=|1,1\rangle$ inversion doublet are selectively ionized. Mass-selective detection of the parent ions is performed in a short linear time-of-flight setup. The ion signal is proportional to the density of neutral ammonia molecules in the laser interaction region.

In Fig. 3 the measured time-of-flight (TOF) distributions for ammonia molecules over the $52.8 \mathrm{~cm}$ distance from the exit of the decelerator to the laser detection region $(33 \mathrm{~cm}$ behind the buncher) are shown for various voltages on the buncher. The vertical scale is the same for all measurements, and the measurements are given an offset for clarity. With the buncher switched on for $239 \mu$ s, i.e., when two bunching stages are used, a longitudinal spatial focus is obtained with voltages of +5.75 and $-5.75 \mathrm{kV}$ on the buncher. From the observed width of the TOF distribution a longitudinal spatial focus of about $2.0 \mathrm{~mm}$ is deduced; the magnification of the package exiting the decelerator with about a factor of 2 is what is expected with the thin-lens approximation for this geometry. With higher voltages, the beam is overfocused, resulting in a broader TOF distribution. In the inset, the measured TOF distributions recorded $4.5 \mathrm{~cm}$ behind the buncher $(24.3 \mathrm{~cm}$ from the exit of the

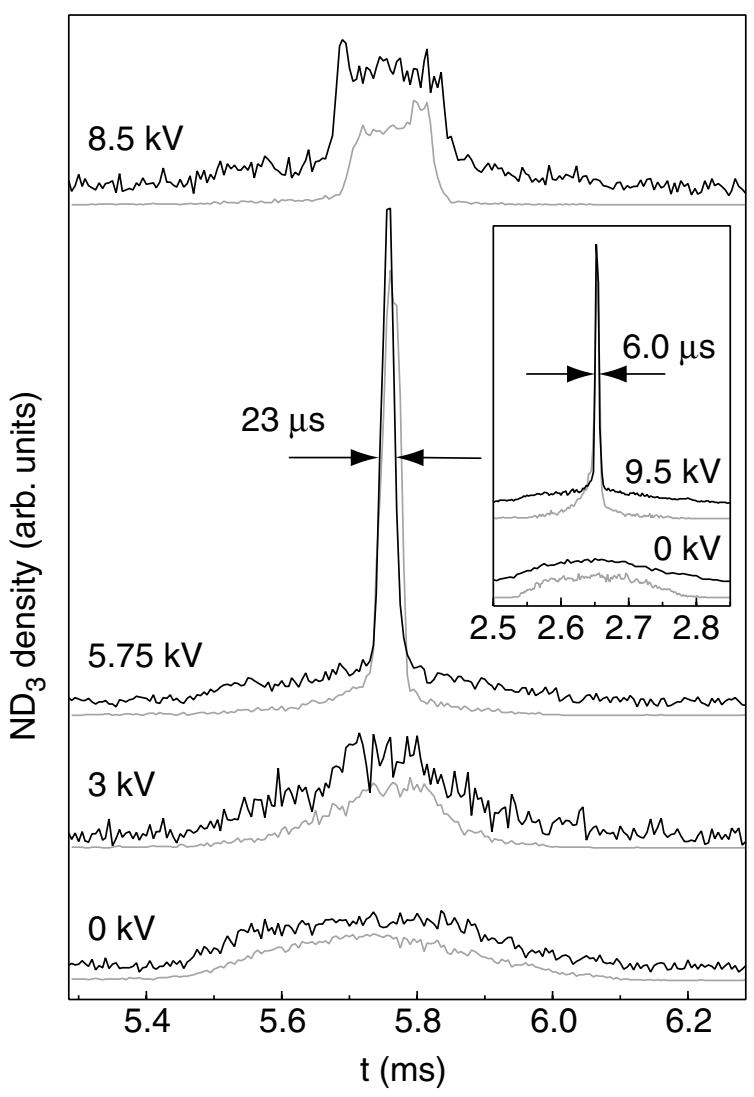

FIG. 3. Density of ammonia molecules $33 \mathrm{~cm}$ behind the buncher as a function of time (at $t=0 \mathrm{~ms}$ the synchronous molecule is at the exit of the decelerator) for different voltages on the buncher, using two bunching stages. In the inset similar measurements recorded at a $4.5 \mathrm{~cm}$ distance behind the buncher, using three bunching stages, are shown. The results of trajectory calculations through the entire setup are shown underneath the experimental data. 


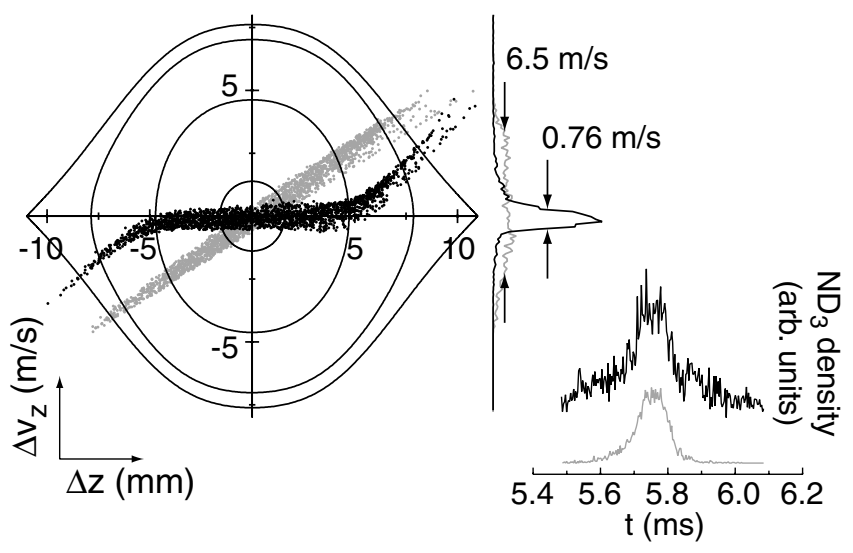

FIG. 4. Calculated longitudinal phase-space distribution at the moment that the buncher is switched on $(t=1.743 \mathrm{~ms})$ and off $(t=1.982 \mathrm{~ms})$ with voltages of +3.8 and $-3.8 \mathrm{kV}$. The projections of the longitudinal velocity distributions on the $v_{z}$ axis are given for both cases. On the right hand side, the measured (upper trace) and calculated (lower trace) TOF distributions $33 \mathrm{~cm}$ behind the buncher are shown.

decelerator) and using three bunching stages are shown. A considerably tighter longitudinal spatial focus is now obtained with voltages of +9.5 and $-9.5 \mathrm{kV}$ on the buncher. The observed $6.0 \mu$ s width of the TOF distribution corresponds to a longitudinal spatial focus of approximately $0.5 \mathrm{~mm}$; the calculated phase-space distributions for this particular situation are the ones shown already in Fig. 2. The observed TOF distributions are quantitatively reproduced in trajectory calculations, shown underneath the experimental data.

It is evident from the description of the operation principle of the buncher that it is also possible to rotate the bunch such that an elongated horizontal distribution is formed in phase space, i.e., that a focus is created in velocity space. The optimum settings for longitudinal cooling of the molecular beam follow directly from the experimentally determined settings for spatial focusing. In Fig. 4 the calculated longitudinal phase-space distribution is shown at the moment that the buncher is switched on and off. In this case two bunching stages are used, and voltages of +3.8 and $-3.8 \mathrm{kV}$ are applied. The projection of the phase-space distribution onto the vertical axis after bunch rotation gives a longitudinal velocity distribution with a full width at half maximum of $0.76 \mathrm{~m} / \mathrm{s}$. This corresponds to a record-low longitudinal temperature of our molecular beam of $250 \mu \mathrm{K}$. The measured and calculated TOF distributions $33 \mathrm{~cm}$ behind the buncher are shown in the figure as well.

In conclusion, we have demonstrated a buncher for longitudinal focusing and cooling of a beam of neutral polar molecules. Tighter spatial focusing and/or further longitudinal cooling can be achieved by properly scaling and positioning the buncher. When this buncher is used in combination with electrostatic multipole lenses, full six- dimensional phase-space matching of one element onto another, for instance, of a molecular beam exiting a decelerator onto a trap or storage ring, is possible.

This work is part of the research program of the "Stichting voor Fundamenteel Onderzoek der Materie (FOM)," which is financially supported by the "Nederlandse Organisatie voor Wetenschappelijk Onderzoek (NWO)." The research of R. T. J. has been made possible by the Royal Netherlands Academy of Arts and Sciences.

[1] J. M. Doyle and B. Friedrich, Nature (London) 401, 749 (1999).

[2] C. J. Williams and P. S. Julienne, Science 287, 986 (2000).

[3] L. Santos, G. V. Shlyapnikov, P. Zoller, and M. Lewenstein, Phys. Rev. Lett. 85, 1791 (2000).

[4] M. A. Baranov, M.S. Mar'enko, Val.S. Rychkov, and G. V. Shlyapnikov, cond-mat/0109437.

[5] D. DeMille, Phys. Rev. Lett. 88, 067901 (2002).

[6] H. L. Bethlem, G. Berden, and G. Meijer, Phys. Rev. Lett. 83, 1558 (1999).

[7] H. L. Bethlem, G. Berden, A. J.A. van Roij, F.M.H. Crompvoets, and G. Meijer, Phys. Rev. Lett. 84, 5744 (2000).

[8] H.L. Bethlem, A. J.A. van Roij, R. T. Jongma, and G. Meijer, Phys. Rev. Lett. 88, 133003 (2002).

[9] W. Schöllkopf and J.P. Toennies, Science 266, 1345 (1994).

[10] M. Arndt, O. Nairz, J. Vos-Andreae, C. Keller, G. van der Zouw, and A. Zeilinger, Nature (London) 401, 680 (1999).

[11] K. Burnett, P. S. Julienne, P. D. Lett, E. Tiesinga, and C. J. Williams, Nature (London) 416, 225 (2002).

[12] Jue Wang, V.A. Shamamian, B.R. Thomas, J. M. Wilkinson, J. Riley, C. F. Giese, and W. R. Gentry, Phys. Rev. Lett. 60, 696 (1988).

[13] F. M. H. Crompvoets, H. L. Bethlem, R. T. Jongma, and G. Meijer, Nature (London) 411, 174 (2001).

[14] H. L. Bethlem, G. Berden, F. M. H. Crompvoets, R. T. Jongma, A.J.A. van Roij, and G. Meijer, Nature (London) 406, 491 (2000).

[15] H. L. Bethlem, F. M. H. Crompvoets, R. T. Jongma, S. Y. T. van de Meerakker, and G. Meijer, Phys. Rev. A 65, 053416 (2002).

[16] W. Ketterle and D.E. Pritchard, Phys. Rev. A 46, 4051 (1992).

[17] S. Humphries, Jr., Charged Particle Beams (John Wiley \& Sons, Inc., New York, 1990).

[18] S. Chu, J.E. Bjorkholm, A. Ashkin, J.P. Gordon, and L. W. Hollberg, Opt. Lett. 11, 73 (1986).

[19] H. Ammann and N. Christensen, Phys. Rev. Lett. 78, 2088 (1997).

[20] J. Summhammer, L. Niel, and H. Rauch, Z. Phys. B 62, 269 (1986).

[21] E. Maréchal, S. Guibal, J.-L. Bossennec, R. Barbé, J.-C. Keller, and O. Gorceix, Phys. Rev. A 59, 4636 (1999).

[22] S. H. Myrskog, J. K. Fox, H. S. Moon, J. B. Kim, and A. M. Steinberg, Phys. Rev. A 61, 053412 (2000). 